PROCEEDINGS OF THE

AMERICAN MATHEMATICAL SOCIETY

Volume 129, Number 10, Pages 2897-2905

S 0002-9939(01)05559-9

Article electronically published on March 15, 2001

\title{
CANONICAL MAPPINGS FOR POLYNOMIALS AND HOLOMORPHIC FUNCTIONS ON BANACH SPACES
}

\author{
SEÁN DINEEN
}

(Communicated by Steven R. Bell)

\begin{abstract}
We obtain functional representations for the canonical mapping into the bidual for spaces of holomorphic functions on certain Banach spaces.
\end{abstract}

\section{INTRODUCTION}

If $E$ is a locally convex space with strong dual $E_{\beta}^{\prime}$, then the canonical mapping $J_{E}$ into the bidual $E^{\prime \prime}:=\left(E_{\beta}^{\prime}\right)_{\beta}^{\prime}$ is obtained by letting $\left(J_{E} x\right)(\phi)=\phi(x)$ for $x \in$ $E$. The topology on $\left(E_{\beta}^{\prime}\right)_{\beta}^{\prime}$ is called the bidual topology. When the bounded and equicontinuous subsets of $E_{\beta}^{\prime}$ coincide (i.e. if $E$ is infrabarrelled or quasibarrelled) then $J_{E}$ is an isomorphism onto its range and if, in addition, $J_{E}$ is surjective, then $E$ is reflexive. Banach spaces are infrabarrelled and, in this case, $J_{E}$ is an isometric embedding when $E_{\beta}^{\prime}$ and $E^{\prime \prime}$ are endowed with the usual norms of uniform convergence on the unit ball.

In this abstract setting the bidual and the canonical image are realised as spaces of linear functions on spaces which are themselves rather abstractly defined. In complex function theory over infinite dimensional spaces we study $\mathcal{H}(E)$, the holomorphic functions on the locally convex space $E$, and it is useful to obtain concrete representations for $J_{\mathcal{H}(E)}, J_{\mathcal{H}(E)}(\mathcal{H}(E))$ and $\mathcal{H}(E)^{\prime \prime}(\mathcal{H}(E)$ is, of course, endowed with some locally convex space structure). The analogous problem for polynomials on Banach spaces, where Banach space methods suffice, has been extensively studied over the last twenty years, and relatively recently the same question for holomorphic functions of bounded type on a Banach space, where Fréchet spaces are involved, has been investigated. We refer to [5] and 8] for background information on infinite dimensional holomorphy and locally convex spaces respectively.

\section{Polynomials And holomorphic FunCtions OF BOUNDED TYPE}

Let $E$ denote a Banach space over $\mathbb{C}$. For each positive integer $n$ let $\mathcal{P}\left({ }^{n} E\right)$ (respectively $\mathcal{P}_{\omega}\left({ }^{n} E\right), \mathcal{P}_{\omega^{*}}\left({ }^{n} E^{\prime \prime}\right)$ ) denote the space of all continuous $n$-homogeneous polynomials on $E$ (respectively all $n$-homogeneous polynomials on $E$ which are weakly (or $\sigma\left(E, E^{\prime}\right)$ ) continuous on bounded sets, all $n$-homogeneous polynomials on $E^{\prime \prime}$ which are weak* (or $\sigma\left(E^{\prime \prime}, E^{\prime}\right)$ ) continuous on bounded sets). We identify

Received by the editors January 6, 1999 and, in revised form, February 2, 2000.

2000 Mathematics Subject Classification. Primary 46G20; Secondary 46G25. 
$\mathcal{P}\left({ }^{0} E\right)$ with the space of constant $\mathbb{C}$-valued mappings on $E$. Clearly $\mathcal{P}_{\omega}\left({ }^{n} E\right) \subseteq$ $\mathcal{P}\left({ }^{n} E\right)$ and $\mathcal{P}_{\omega^{*}}\left({ }^{n} E^{\prime \prime}\right) \subseteq \mathcal{P}\left({ }^{n} E^{\prime \prime}\right)$. Let $B$ denote the open unit ball in $E$. We endow $\mathcal{P}\left({ }^{n} E\right)$ with the topology of uniform convergence over $B$, i.e. with the topology generated by the norm $\|\cdot\|_{B}$ and give $\mathcal{P}_{\omega}\left({ }^{n} E\right)$ the induced topology. The space $\mathcal{P}\left({ }^{n} E^{\prime \prime}\right)$ is given the topology of uniform convergence over the unit ball of $E^{\prime \prime}, B^{\prime \prime}$, and $\mathcal{P}_{\omega^{*}}\left({ }^{n} E^{\prime \prime}\right)$ is given the induced topology.

Proposition 1. If $E$ is a Banach space and $E^{\prime \prime}$ has the approximation property and the Radon-Nikodym property, then

$$
\begin{gathered}
J_{\mathcal{P}_{\omega}\left({ }^{n} E\right)}\left(\mathcal{P}_{\omega}\left({ }^{n} E\right)\right)=\mathcal{P}_{\omega^{*}}\left({ }^{n} E^{\prime \prime}\right), \\
\mathcal{P}_{\omega}\left({ }^{n} E\right)^{\prime \prime}=\mathcal{P}\left({ }^{n} E^{\prime \prime}\right),
\end{gathered}
$$

and

$$
\left(J_{\mathcal{P}_{\omega}\left({ }^{n} E\right)}(P)\right)\left(x^{\prime \prime}\right)=\lim _{\alpha} P\left(x_{\alpha}\right)
$$

for all $P \in \mathcal{P}_{\omega}\left({ }^{n} E\right)$ and $x^{\prime \prime} \in E^{\prime \prime}$ where $\left(x_{\alpha}\right)_{\alpha}$ is any bounded net in $E$ satisfying $\lim _{\alpha} J_{E}\left(x_{\alpha}\right)=x^{\prime \prime}$ in the $\sigma\left(E^{\prime \prime}, E^{\prime}\right)$ topology on $E^{\prime \prime}$.

Remark. The above proposition is the culmination of research by a number of different authors; we refer to [5] for details. The Radon-Nikodým Property on $E^{\prime \prime}$ can be replaced by the weaker hypothesis that $l_{1}$ does not belong to the completed $n$-fold symmetric injective (or $\epsilon$ ) tensor product of $E^{\prime}$ (see [3] and [4). If $E$ is a $Q$-reflexive Banach space (see 2] and 7]) and $E^{\prime \prime}$ has the approximation property, then $\mathcal{P}\left({ }^{n} E\right)=\mathcal{P}_{\omega}\left({ }^{n} E\right)$ and $\mathcal{P}_{\omega}\left({ }^{n} E\right)^{\prime \prime}$ is isomorphic to $\mathcal{P}\left({ }^{n} E^{\prime \prime}\right)$ but it is not known if the spaces are isometrically isomorphic under this isomorphism.

Holomorphic functions on locally convex spaces can be introduced in many different ways (see 5, Chapter 3]) but for the purposes of this paper it is convenient to use Taylor series expansions. If $U$ is an open subset of a Banach space and $f: U \longrightarrow \mathbb{C}$, then $f$ is holomorphic on $U$ if, for each $x_{0} \in U$, there exists a sequence $\left(P_{n, x_{0}}\right)_{n=0}^{\infty}, P_{n, x_{0}} \in \mathcal{P}\left({ }^{n} E\right)$ for all $n$, such that

$$
f\left(x_{0}+y\right)=\sum_{n=0}^{\infty} P_{n, x_{0}}(y)
$$

for all $y$ in some neighbourhood of the origin. We call (1) the Taylor series expansion of $f$ about $x_{0}$ and use the notation $\frac{\widehat{d} f^{n}\left(x_{0}\right)}{n !}=P_{n, x_{0}}$ for all $n$. Let $\mathcal{H}(U)$ denote the space of all holomorphic functions on $U$. Let $B_{r}$ denote the open ball of radius $r$, $0<r \leq \infty$, in $E$ centered at the origin. Let

$$
\mathcal{H}_{b}\left(B_{r}\right):=\left\{f \in \mathcal{H}\left(B_{r}\right) ;\|f\|_{B_{\rho}}<\infty \text { for all } \rho<r\right\} .
$$

The space $\mathcal{H}_{b}\left(B_{r}\right)$, endowed with the topology generated by the semi-norms, $\|\cdot\|_{\rho}$, $0<\rho<r$, is a Fréchet space and functions in $\mathcal{H}_{b}\left(B_{r}\right)$ are called holomorphic functions of bounded type. We also let

$$
\mathcal{H}_{\omega, b}\left(B_{r}\right):=\left\{f \in \mathcal{H}_{b}\left(B_{r}\right) ; \frac{\widehat{d}^{n} f\left(x_{0}\right)}{n !} \in \mathcal{P}_{\omega}\left({ }^{n} E\right) \text { for all } n\right\}
$$

and

$$
\mathcal{H}_{\omega^{*}, b}\left(B_{r}^{\prime \prime}\right):=\left\{f \in \mathcal{H}_{b}\left(B_{r}^{\prime \prime}\right) ; \frac{\widehat{d}^{n} f\left(x_{0}\right)}{n !} \in \mathcal{P}_{\omega^{*}}\left({ }^{n} E^{\prime \prime}\right) \text { for all } n\right\}
$$


where $B_{r}^{\prime \prime}$ is the ball of radius $r$ in $E^{\prime \prime}$ centered at the origin. We endow $\mathcal{H}_{\omega, b}\left(B_{r}\right)$ and $\mathcal{H}_{\omega^{*}, b}\left(B_{r}^{\prime \prime}\right)$ with the Fréchet space topologies induced by $\mathcal{H}_{b}\left(B_{r}\right)$ and $\mathcal{H}_{b}\left(B_{r}^{\prime \prime}\right)$ respectively. The functions in $\mathcal{H}_{\omega, b}\left(B_{r}\right)$ and $\mathcal{H}_{\omega^{*}, b}\left(B_{r}^{\prime \prime}\right)$ are the holomorphic functions on $B_{r}$ and $B_{r}^{\prime \prime}$ which are weakly (respectively weak ${ }^{*}$ ) continuous on bounded sets. The following is the analogue of Proposition 1 for holomorphic functions of bounded type $([\underline{6})$.

Proposition 2. If $E$ is a Banach space, $E^{\prime \prime}$ has the approximation property and the Radon-Nikodym property and $0<r \leq \infty$, then

$$
\begin{gathered}
J_{\mathcal{H}_{\omega, b}\left(B_{r}\right)}=\sum_{n=o}^{\infty} J_{\mathcal{P}_{\omega}\left({ }^{n} E\right)}, \\
J_{\mathcal{H}_{\omega, b}\left(B_{r}\right)}\left(\left(\mathcal{H}_{\omega, b}\left(B_{r}\right)\right)=\mathcal{H}_{\omega^{*}, b}\left(B_{r}^{\prime \prime}\right),\right. \\
\mathcal{H}_{\omega, b}\left(B_{r}\right)^{\prime \prime}=\mathcal{H}_{b}\left(B_{r}^{\prime \prime}\right) .
\end{gathered}
$$

\section{Holomorphic FunCtions on BANACH SPACES}

To obtain results, similar to those in the previous section, for holomorphic functions we must discuss various topologies on $\mathcal{H}(U)$. One of our main techniques is the application of $\mathcal{S}$-absolute decompositions of locally convex spaces. Let

$$
\mathcal{S}:=\left\{\left(\alpha_{n}\right)_{n} ; \alpha_{n} \in \mathbb{C}, \limsup _{n}\left|\alpha_{n}\right|^{1 / n} \leq 1\right\} .
$$

Definition 1. A Schauder decomposition $\left\{E_{n}\right\}_{n}$ of a locally convex space $E$ is an $\mathcal{S}$-absolute decomposition if the following two properties are satisfied:

(a) If $x=\sum_{n=1}^{\infty} x_{n} \in E, x_{n} \in E_{n}$ for all $n$ and $\left(\alpha_{n}\right)_{n} \in \mathcal{S}$, then $\sum_{n=1}^{\infty} \alpha_{n} x_{n} \in E$.

(b) If $p$ is a continuous semi-norm on $E$ and $\left(\alpha_{n}\right)_{n} \in \mathcal{S}$, then

$$
q\left(\sum_{n=1}^{\infty} x_{n}\right):=\sum_{n=1}^{\infty}\left|\alpha_{n}\right| p\left(x_{n}\right)
$$

defines a continuous semi-norm on $E$.

If $c s(E)$ denotes the set of all continuous semi-norms on $E$ and $\left\{E_{n}\right\}$ is an $\mathcal{S}$-absolute decomposition for $E$, then

$$
\begin{aligned}
E & =\left\{\sum_{n=1}^{\infty} x_{n} ; \sum_{n=1}^{\infty}\left|\alpha_{n}\right| p\left(x_{n}\right)<\infty \text { for all } p \in \operatorname{cs}(E) \text { and all }\left(\alpha_{n}\right)_{n} \in \mathcal{S}\right\} \\
& =\left\{\sum_{n=1}^{\infty} x_{n} ; \sup _{n}\left|\alpha_{n}\right| p\left(x_{n}\right)<\infty \text { for all } p \in \operatorname{cs}(E) \text { and all }\left(\alpha_{n}\right)_{n} \in \mathcal{S}\right\} \\
& =\left\{\sum_{n=1}^{\infty} x_{n} ; \lim _{n}\left|\alpha_{n}\right| p\left(x_{n}\right)=0 \text { for all } p \in \operatorname{cs}(E) \text { and all }\left(\alpha_{n}\right)_{n} \in \mathcal{S}\right\} .
\end{aligned}
$$

Hence the topology on $E$ is generated by either of the following collections:

$$
\begin{aligned}
& \left\{\sum_{n=1}^{\infty} x_{n} \longrightarrow \sum_{n=1}^{\infty}\left|\alpha_{n}\right| p\left(x_{n}\right)\right\}_{p \in c s(E),\left(\alpha_{n}\right)_{n} \in \mathcal{S}} \\
& \left\{\sum_{n=1}^{\infty} x_{n} \longrightarrow \sup _{n}\left|\alpha_{n}\right| p\left(x_{n}\right)\right\}_{p \in c s(E),\left(\alpha_{n}\right)_{n} \in \mathcal{S}} .
\end{aligned}
$$


As a result of these representations we can switch, in spaces with $\mathcal{S}$-absolute decomposition, from supremums to summations in presenting either fundamental systems of semi-norms or bounded sets. If $\left\{E_{n}\right\}_{n}$ is an $\mathcal{S}$-absolute decomposition of $E$, then $\left\{\left(E_{n}^{\prime}\right)_{\beta}\right\}_{n}$ is an $\mathcal{S}$-absolute decomposition of $E_{\beta}^{\prime}$ ([5. Proposition 3.35]). If $U$ is an open subset of a Banach space we let $\tau_{0}$ denote the compact open topology on $\mathcal{C}(U)$, the space of continuous $\mathbb{C}$-valued functions on $U$. If $\mathcal{F}(U)$ is a linear subspace of $\mathcal{C}(U)$ and $p$ is a semi-norm on $\mathcal{F}(U)$ we say that $p$ is ported by the compact subset $K$ of $U$ if for every $V$ open, $K \subset V \subset U$, there exists $C(V)>0$ such that

$$
p(f) \leq C(V)\|f\|_{V}
$$

for all $f$ in $\mathcal{F}(U)$. Let $\tau_{\omega}(\mathcal{F}(U))$ denote the locally convex topology on $\mathcal{F}(U)$ generated by the semi-norms on $\mathcal{F}(U)$ ported by compact subsets of $U$. Clearly $\tau_{\omega}(\mathcal{F}(U)) \geq \tau_{0}$.

Example. Let $E$ denote an infinite dimensional separable Banach space. The $\tau_{0}$ bounded subsets of $\mathcal{C}(E)$ are locally bounded and hence $\tau_{\omega}(\mathcal{C}(E))$ bounded. Hence $\tau_{0}$ and $\tau_{\omega}(\mathcal{C}(E))$ define the same bounded subsets of $(\mathcal{C}(E))$. By a theorem of L. Nachbin [10] and T. Shirota [11] the space $\left(\mathcal{C}(E), \tau_{0}\right)$ is bornological. Since $\tau_{\omega}(\mathcal{C}(E)) \geq \tau_{0}$ this implies $\tau_{\omega}(\mathcal{C}(E))=\tau_{0}$. On the other hand it is easily seen that $\tau_{0} \neq \tau_{\omega}$ on $\mathcal{H}(E)$. The above shows that $\left.\tau_{\omega}(\mathcal{C}(E))\right|_{\mathcal{H}(E)} \neq \tau_{\omega}(\mathcal{H}(E))$. We let $\mathcal{H}_{\omega}(U)$ denote the subspace of $\mathcal{H}(U), U$ an open subset of a Banach space, consisting of all functions which are locally (with respect to the norm) uniformly weakly continuous. If $U$ is balanced, then the following lemma, which is easily proved using Taylor series expansions at the origin, provides an alternative description of this space.

Lemma 1. If $U$ is a balanced open subset of a Banach space and $f \in \mathcal{H}(U)$, then $f \in \mathcal{H}_{\omega}(U)$ if and only if $\frac{\widehat{d}^{n} f\left(x_{0}\right)}{n !} \in \mathcal{P}_{\omega}\left({ }^{n} E\right)$ for all $n$.

The Taylor series expansion at the origin gives rise to $\mathcal{S}$-absolute decompositions for various spaces of holomorphic functions and we have the following examples.

Let $U$ be a balanced open subset of the Banach space $E$ :

(a) $\left(\mathcal{P}\left({ }^{n} E\right),\|\cdot\|_{B}\right)_{n=0}^{\infty}$ is an $\mathcal{S}$-absolute decomposition for $\left(\mathcal{H}(U), \tau_{\omega}\right)$ and $\left(\mathcal{H}_{b}(U)\right.$.

(b) $\left(\mathcal{P}\left({ }^{n} E\right), \tau_{0}\right)_{n=0}^{\infty}$ is an $\mathcal{S}$-absolute decomposition for $\left(\mathcal{H}(U), \tau_{0}\right)$.

(c) $\left(\mathcal{P}_{\omega}\left({ }^{n} E\right),\|\cdot\|_{B}\right)_{n=0}^{\infty}$ is an $\mathcal{S}$-absolute decomposition for $\left(\mathcal{H}_{\omega}(U), \tau_{\omega}\right)$.

(d) If $\left(\mathcal{H}_{\omega^{*}}(U), \tau_{\omega}\right)$ is the subspace of $\left(\mathcal{H}(U), \tau_{\omega}\right), U$ balanced open in $E^{\prime \prime}$, consisting of functions which are locally (with respect to the norm) weak* continuous, then $\left(\mathcal{P}_{\omega^{*}}\left({ }^{n} E^{\prime \prime}\right),\|\cdot\|_{B^{\prime \prime}}\right)_{n=0}^{\infty}$ is an $\mathcal{S}$-absolute decomposition for $\left(\mathcal{H}_{\omega^{*}}(U), \tau_{\omega}\right)$.

The $\tau_{\omega}$ topology on $\mathcal{H}(U), U$ balanced, is generated by the semi-norms

$$
p_{K,\left(\beta_{n}\right)_{n}}\left(\sum_{n=0}^{\infty} \frac{\widehat{d}^{n} f\left(x_{0}\right)}{n !}\right)=\sum_{n=0}^{\infty}\left\|\frac{\widehat{d}^{n} f\left(x_{0}\right)}{n !}\right\|_{K+\beta_{n} B}
$$

where $K$ ranges over the compact balanced subsets of $U$ and $\left(\beta_{n}\right)_{n}$ over $c_{0}$ (see [5] for further details).

Proposition 3. If $U$ is a balanced open subset of a Banach space E, then

$$
\left.\tau_{\omega}(\mathcal{H}(U))\right|_{\mathcal{H}_{\omega}(U)}=\tau_{\omega}\left(\mathcal{H}_{\omega}(U)\right)
$$

Proof. Since $\left(\mathcal{P}\left({ }^{n} E\right),\|\cdot\|_{B}\right)_{n=0}^{\infty}$ is an $\mathcal{S}$-absolute decomposition for $\mathcal{H}_{\omega}(U)$ endowed with the $\tau_{\omega}\left(\mathcal{H}_{\omega}(U)\right)$ topology, the proof given in [5, pp. 211-212] for $\left(\mathcal{H}(U), \tau_{\omega}\right)$ can 
be modified to show that the $\tau_{\omega}\left(\mathcal{H}_{\omega}(U)\right)$ topology on $\mathcal{H}_{\omega}(U)$ is generated by all the semi-norms satisfying (5). This completes the proof.

Since there is now no danger of ambiguity we write $\tau_{\omega}$ in place of $\tau_{\omega}\left(\mathcal{H}_{\omega}(U)\right)$ and $\tau_{\omega}(\mathcal{H}(U))$. Our aim is to show that $\left(\mathcal{H}_{\omega}(U), \tau_{\omega}\right)$ is ultrabornological, and hence bornological and infrabarrelled, when $E$ is a Banach space with the bounded approximation property. The result is known to hold for $\left(\mathcal{H}(U), \tau_{\omega}\right)$ but the proof is rather technical (see [5, Chapter 4]). However the proof can be modified to obtain the result that we require for $\mathcal{H}_{\omega}(U)$.

Proposition 4. If $U$ is a balanced open subset of a separable Banach space with the bounded approximation property $E$, then $\left(\mathcal{H}_{\omega}(U), \tau_{\omega}\right)$ is ultrabornological.

Proof. Let $\mathcal{V}:=\left(V_{n}\right)_{n}$ denote a countable open cover of $U$ and let

$$
\mathcal{H}_{\mathcal{V}, \omega}:=\left\{f \in \mathcal{H}_{\omega}(U) ;\|f\|_{V_{n}}<\infty \text { for all } n\right\} .
$$

We endow $\mathcal{V}:=\left(V_{n}\right)_{n}$ with the topology generated by the sequence of semi-norms

$$
f \longrightarrow\|f\|_{V_{n}}, f \in \mathcal{H}_{\omega}(U), n=1,2, \ldots
$$

Since $\mathcal{P}_{\omega}\left({ }^{n} E\right)$ is a Banach space for all $n$ the space $\mathcal{H}_{\mathcal{V}, \omega}$ is a Fréchet space. It is easily seen that $\mathcal{H}_{\omega}(U)=\bigcup_{\mathcal{V}} \mathcal{H}_{\mathcal{V}, \omega}$. Let $\tau_{\delta}\left(\mathcal{H}_{\omega}(U)\right)$ denote the inductive limit topology on $\mathcal{H}_{\omega}(U)$ arising from $\lim _{\mathcal{\nu}} \mathcal{H}_{\mathcal{V}, \omega}$. Since $\mathcal{H}_{\mathcal{V}, \omega}$ is a Fréchet space for all $\mathcal{V}$ it follows that $\tau_{\delta}\left(\mathcal{H}_{\omega}(U)\right)$ is an ultrabornological topology. Moreover, since the $\tau_{\delta}\left(\mathcal{H}_{\omega}(U)\right)$-bounded and the $\tau_{\omega}$-bounded subsets of $\mathcal{H}_{\omega}(U)$ coincide, the topology $\tau_{\delta}\left(\mathcal{H}_{\omega}(U)\right)$ is the bornological topology associated with $\tau_{\omega}$ (we refer to $[5$ for further details). To complete the proof it suffices to show that all Banach-valued $\tau_{\delta}\left(\mathcal{H}_{\omega}(U)\right)$ continuous mappings on $\mathcal{H}_{\omega}(U)$ are $\tau_{\omega}$ continuous. We first suppose that $E$ has a Schauder basis $\left(e_{n}\right)_{n}$. Let $\pi_{n}$ denote the canonical projection from $E$ onto the span of the first $n$-coordinates and let $\pi^{n}=I d-\pi_{n}$. The proof of the corresponding result given in [5, Chapter 4] extends to $\mathcal{H}_{\omega}(U)$ once we have established that the following results hold:

(a) if $n$ is a positive integer, $P \in \mathcal{P}_{\omega}\left({ }^{n} E\right), 0 \leq m<\infty$ and $0 \leq j \leq n$, then the mapping

$$
P_{m, j}: x \in E \longrightarrow \check{P}\left(\left(\pi_{m}(x)\right)^{j},\left(\pi^{m}(x)\right)^{n-j}\right)
$$

belongs to $\mathcal{P}_{\omega}\left({ }^{n} E\right)$ ( $\check{P}$ is the unique symmetric $n$-linear form associated with $P$ ),

(b) if $\left(f_{n}\right)_{n} \subset \mathcal{H}_{\omega}(U)$ and $\sum_{n=0}^{\infty}\left|\frac{\widehat{d}^{n} f_{n}(0)}{n !}(x)\right|<\infty$ for all $x \in U$, then $\sum_{n=0}^{\infty} \frac{\widehat{d}^{n} f_{n}(0)}{n !}$ $\in \mathcal{H}_{\omega}(U)$.

To establish (a) we note that $E$ has the approximation property and hence $\mathcal{P}_{\omega}\left({ }^{n} E\right)$ coincides with the Banach space of approximable $n$-homogeneous polynomials. By density we may thus suppose that $P=\varphi^{n}$ where $\varphi \in E^{\prime}$. In this case (6) reduces to the mapping

$$
x \in E \longrightarrow \varphi\left(\pi_{m}(x)\right)^{j} \varphi\left(\pi^{m}(x)\right)^{n-j} .
$$

Since the mappings

$$
x \longrightarrow \varphi\left(\pi_{m}(x)\right)^{j} \text { and } x \longrightarrow \varphi\left(\pi^{m}(x)\right)^{n-j}
$$

belong to $\mathcal{P}_{A}\left({ }^{j} E\right)$ and $\mathcal{P}_{A}\left({ }^{n-j} E\right)$, respectively, and the space of approximable polynomials is an algebra, (a) holds for all $\varphi^{n}$ and hence for all $P \in \mathcal{P}_{\omega}\left({ }^{n} E\right)$. If the sequence $\left(f_{n}\right)_{n}$ satisfies the hypothesis in (b), then by [5. Example 3.8] 
$\left.\sum_{n=0}^{\infty} \frac{\widehat{d}^{n} f_{n}(0)}{n !}\right) \in \mathcal{H}(U)$ and, by Lemma 1, (b) holds. This completes the proof when $E$ has a Schauder basis.

If $E$ is separable and has the bounded approximation property, then $E$ is isomorphic to a complemented subspace of a Banach space with a Schauder basis. The equality $\tau_{\delta}\left(\mathcal{H}_{\omega}(U)\right)=\tau_{\omega}\left(\mathcal{H}_{\omega}(U)\right)$ passes to complemented subspaces (see the proof of [5, Lemma 4.17]) once it is observed that the extension of $f \in \mathcal{H}_{\omega}(U)$ to $\tilde{f}$ on $U \times F, F$ an arbitrary Banach space, given by $\tilde{f}(x+y):=f(x)$ is holomorphic. This completes the proof.

We next obtain a (Taylor) series representation of the bidual and then use this to obtain a functional representation. For $r>0$ let $\mathcal{K}\left(B_{r}\right)$ denote the set of all compact convex balanced subsets of $B_{r}$. If $K \in \mathcal{K}\left(B_{r}\right)$, then $J_{E}(K) \in \mathcal{K}\left(B_{r}^{\prime \prime}\right)$ and, moreover, for $\epsilon>0$

$$
\left(\mathcal{K}+B_{\epsilon}\right)^{\circ \circ}=\mathcal{K}^{\circ \circ}+B_{\epsilon}^{\circ \circ}=J_{E}(\mathcal{K})+B_{\epsilon}^{\circ \circ} .
$$

If now $E^{\prime \prime}$ has the approximation property and the Radon-Nikodým, then since these two properties are invariant under renormings, Proposition 1 and (6) imply that

$$
\left\{P \in \mathcal{P}_{\omega}\left({ }^{n} E\right) ;\|P\|_{K+B_{\epsilon}} \leq 1\right\}^{\circ 0}=\left\{P \in \mathcal{P}\left({ }^{n} E^{\prime \prime}\right) ;\|P\|_{J_{E}(K)+B_{\epsilon}^{\prime \prime}} \leq 1\right\}
$$

and

$$
J_{\mathcal{P}_{\omega}\left({ }^{n} E\right)}\left(\left\{P \in \mathcal{P}_{\omega}\left({ }^{n} E\right) ;\|P\|_{K+B_{\epsilon}} \leq 1\right\}\right)=\left\{P \in \mathcal{P}_{\omega^{*}}\left({ }^{n} E^{\prime \prime}\right) ;\|P\|_{J_{E}(K)+B_{\epsilon}^{\prime \prime}} \leq 1\right\}
$$

for all $K \in \mathcal{K}\left(B_{r}\right)$ and all $\epsilon>0$.

Proposition 5. If $E$ is a Banach space with the bounded approximation property and $E^{\prime \prime}$ has the approximation property and the Radon-Nikodym property, then the following hold:

$$
\begin{aligned}
\left(\mathcal{H}_{\omega}\left(B_{r}\right), \tau_{\omega}\right)^{\prime \prime}= & \left\{\sum_{n=0}^{\infty} P_{n} ; P_{n} \in \mathcal{P}\left({ }^{n} E^{\prime \prime}\right) \text { and } \sum_{n=0}^{\infty}\left\|P_{n}\right\|_{J_{E}(K)+\beta_{n} B^{\prime \prime}}<\infty\right. \\
& \text { for all } \left.K \in \mathcal{K}\left(B_{r}\right) \text { and all }\left(\beta_{n}\right)_{n} \in c_{0}\right\} .
\end{aligned}
$$

The bidual topology on $\left(\mathcal{H}_{\omega}\left(B_{r}\right), \tau_{\omega}\right)^{\prime \prime}$ is generated by the seminorms

$$
p_{K,\left(\beta_{n}\right)_{n}}\left(\sum_{n=0}^{\infty} P_{n}\right):=\sum_{n=0}^{\infty}\left\|P_{n}\right\|_{J_{E}(K)+\beta_{n} B^{\prime \prime}}
$$

when $K$ ranges over $\mathcal{K}\left(B_{r}\right)$ and $\left(\beta_{n}\right)_{n}$ over $c_{0}$,

$$
J_{\mathcal{H}_{\omega}\left(B_{r}\right)}\left(\mathcal{H}_{\omega}\left(B_{r}\right)\right)=\left\{\sum_{n=0}^{\infty} P_{n} ; P_{n} \in \mathcal{P}_{\omega^{*}}\left({ }^{n} E^{\prime \prime}\right) \text { for all } n \text { and } \sum_{n=0}^{\infty} P_{n} \in \mathcal{H}_{\omega}\left(B_{r}\right)^{\prime \prime}\right\}
$$

and

$$
J_{\mathcal{H}_{\omega}\left(B_{r}\right)}\left(\sum_{n=0}^{\infty} P_{n}\right)=\sum_{n=0}^{\infty} J_{\mathcal{P}_{\omega}\left({ }^{n} E\right)}\left(P_{n}\right)
$$

for all $\sum_{n=0}^{\infty} P_{n} \in \mathcal{H}_{\omega}\left(B_{r}\right)$. 
Proof. Since $\left\{\left(\mathcal{P}_{\omega}\left({ }^{n} E\right),\|\cdot\|_{B}\right)\right\}_{n=0}^{\infty}$ is an $\mathcal{S}$-absolute decomposition for $\left(\mathcal{H}_{\omega}\left(B_{r}\right), \tau_{\omega}\right)$ the sequence $\left\{\left(\mathcal{P}_{\omega}\left({ }^{n} E\right)^{\prime \prime},\|\cdot\|_{B^{\prime \prime}}\right)\right\}_{n=0}^{\infty}$ is an $\mathcal{S}$-absolute decomposition for $\left(\mathcal{H}_{\omega}\left(B_{r}\right)\right.$, $\left.\tau_{\omega}\right)^{\prime \prime}$. As $E$ has the approximation property and the Radon-Nikodým property, [5. Proposition 3.35] implies that $\left\{\left(\mathcal{P}\left({ }^{n} E^{\prime \prime}\right),\|\cdot\|_{B^{\prime \prime}}\right)\right\}_{n=0}^{\infty}$ is an $\mathcal{S}$-absolute decomposition for $\left(\mathcal{H}_{\omega}\left(B_{r}\right), \tau_{\omega}\right)^{\prime \prime}$. Since $E$ has the bounded approximation property Proposition 4 implies that $\left(\mathcal{H}_{\omega}\left(B_{r}\right), \tau_{\omega}\right)$ is infrabarrelled and hence a fundamental neighbourhood system at the origin in $\left(\mathcal{H}_{\omega}\left(B_{r}\right), \tau_{\omega}\right)^{\prime \prime}$ is given by

$$
\left\{\sum_{n=0}^{\infty} P_{n} \in \mathcal{H}_{\omega}\left(B_{r}\right) ; \sum_{n=0}^{\infty}\left\|P_{n}\right\|_{K+\beta_{n} B} \leq 1\right\}^{\circ \circ}
$$

where $K$ ranges over $\mathcal{K}\left(B_{r}\right)$ and $\left(\beta_{n}\right)_{n}$ over $c_{0}$. We are working with an $\mathcal{S}$-absolute decomposition and hence this fundamental neighbourhood system can be replaced by

$$
\left\{\sum_{n=0}^{\infty} P_{n} ; P_{n} \in \mathcal{P}_{\omega}\left({ }^{n} E\right):\left\|P_{n}\right\|_{K+\beta_{n} B} \leq 1 \text { for all } n\right\}^{\circ \circ} .
$$

By (8) this set coincides with

$$
\left\{\sum_{n=0}^{\infty} P_{n} ; P_{n} \in \mathcal{P}\left({ }^{n} E^{\prime \prime}\right),\left\|P_{n}\right\|_{J_{E}(K)+\beta_{n} B^{\prime \prime}} \leq 1\right\}
$$

and once more using the $\mathcal{S}$-absolute decomposition we obtain (10). Proposition 1 and (9) imply (11) and (12) while (13) follows from Proposition 2 and the easily verified identity $J_{E_{1} \times E_{2}} \equiv J_{E_{1}} \times J_{E_{2}}$ valid for any pair of locally convex spaces $E_{1}$ and $E_{2}$. This completes the proof.

To develop a functional representation we require the following result.

Proposition 6. If $P_{n} \in \mathcal{P}\left({ }^{n} E^{\prime \prime}\right)$ for all $n$ and $\sum_{n=0}^{\infty}\left\|P_{n}\right\|_{J_{E}(K)+\beta_{n} B^{\prime \prime}}<\infty$ for all $K \in \mathcal{K}\left(B_{r}\right)$ and all $\left(\beta_{n}\right)_{n} \in c_{0}$, then there exists $\delta>0$ such that

$$
\sum_{n=0}^{\infty}\left\|P_{n}\right\|_{J_{E}(K)+\delta B^{\prime \prime}}<\infty .
$$

Proof. If the result is not true, then there exists $K \in \mathcal{K}\left(B_{r}\right)$ such that for every positive integer $j$

$$
\sum_{n=0}^{\infty}\left\|P_{n}\right\|_{J_{E}(K)+\frac{1}{j} B^{\prime \prime}}=\infty .
$$

Hence we can choose a strictly increasing sequence of positive integers $\left(n_{j}\right)_{j}$ such that

$$
\sum_{n_{j}+1}^{n_{j+1}}\left\|P_{n}\right\|_{J_{E}(K)+\frac{1}{j} B^{\prime \prime}} \geq 1
$$

for all $j$. Let $l_{n}=1$ for $n<n_{2}$ and $l_{n}=\frac{1}{j}$ for $n_{j} \leq n<n_{j+1}, j \geq 2$. Then $\left(l_{n}\right)_{n} \in c_{0}$ and

$$
\sum_{n=0}^{\infty}\left\|P_{n}\right\|_{J_{E}(K)+l_{n} B^{\prime \prime}}=\infty
$$

This contradicts our hypothesis and completes the proof. 
Proposition 6 and (10) may now be combined to prove the following result.

Corollary 1. If $\sum_{n=0}^{\infty} P_{n} \in \mathcal{H}_{\omega}\left(B_{r}\right)^{\prime \prime}$, then there exists an open subset $U$ of $E^{\prime \prime}$, $B_{r}^{\prime \prime} \subset U$, and $f \in \mathcal{H}(U)$ such that $\sum_{n=0}^{\infty} P_{n}$ is the Taylor series expansion of $f$ at the origin in $E^{\prime \prime}$.

This corollary allows us to identify $\mathcal{H}_{\omega}\left(B_{r}\right)^{\prime \prime}$ with the space of holomorphic germs on the closed subset

$$
J_{E}\left(B_{r}\right)=B_{r}^{\prime \prime} \cap J_{E}(E) \text { of } B_{r}^{\prime \prime} .
$$

Definition 2. Let

$$
\mathcal{H}\left(J_{E}\left(B_{r}\right) \subset B_{r}^{\prime \prime}\right):=\bigcup\left\{\mathcal{H}(V) ; J_{E}\left(B_{r}\right) \subset V \subset B_{r}^{\prime \prime}\right\} / \sim
$$

where $f \sim g$ if $f$ and $g$ coincide on a neighbourhood of $J_{E}\left(B_{r}\right)$ in $B_{r}^{\prime \prime}$. Let

$$
\mathcal{H}_{\omega^{*}}\left(J_{E}\left(B_{r}\right)\right):=\left\{f \in \mathcal{H}\left(J_{E}\left(B_{r}\right) \subset B_{r}^{\prime \prime}\right) ; \frac{\widehat{d}^{n} f\left(x_{0}\right)}{n !} \in \mathcal{P}_{\omega^{*}}\left({ }^{n} E^{\prime \prime}\right) \text { for all } n\right\} .
$$

Proposition 7. If $E$ is a Banach space with the bounded approximation property and $E^{\prime \prime}$ has the Radon-Nikodym property and the approximation property, then, for $0<r \leq \infty$,

$$
\mathcal{H}_{\omega}\left(B_{r}\right)^{\prime \prime}=\mathcal{H}\left(J_{E}\left(B_{r}\right) \subset B_{r}^{\prime \prime}\right)
$$

and

$$
J_{\mathcal{H}_{\omega}\left(B_{r}\right)}\left(\mathcal{H}_{\omega}\left(B_{r}\right)\right)=\mathcal{H}_{\omega^{*}}\left(J_{E}\left(B_{r}\right)\right) .
$$

The embedding $J_{\mathcal{H}_{\omega}\left(B_{r}\right)}(f)$ is obtained term by term using local weak uniform continuity of the polynomials in the Taylor series expansion at the origin.

There is however another natural topology that may be placed on any space of germs: the inductive topology. We denote this topology by $\tau_{i}$. By definition

$$
\mathcal{H}\left(J_{E}\left(B_{r}\right) \subset B_{r}^{\prime \prime}\right)=\lim _{\vec{V}}\left(\mathcal{H}(V), \tau_{\delta}\right)
$$

where the inductive limit is taken over all open subsets of $E^{\prime \prime}$ which contain $J_{E}\left(B_{r}\right)$. By (11) $\tau_{i}$ is stronger than the bidual topology on $\mathcal{H}\left(B_{r}\right)^{\prime \prime}$ and by Lemma 1 the two topologies have the same bounded sets, i.e. the locally bounded subsets of $\mathcal{H}(V)$ where $V$ ranges over all open subsets of $B_{r}^{\prime \prime}$ containing $J_{E}\left(B_{r}\right)$. This implies, in particular, that the above representation for $\tau_{i}$ is a regular inductive limit and $\tau_{i}$ is the bornological topology associated with the bidual topology on $\mathcal{H}\left(B_{r}\right)^{\prime \prime}$. We do not know if these topologies coincide and note that the inductive limit is not countable for any infinite dimensional Banach space.

Remarks. (a) If $E$ is a Banach space with the bounded approximation property, $E^{\prime \prime}$ has the approximation property and the Radon-Nikodým property and $\mathcal{P}_{\omega}\left({ }^{n} E\right)=$ $\mathcal{P}\left({ }^{n} E\right)$, then

$$
\left(\mathcal{H}(E), \tau_{\omega}\right)^{\prime \prime}=\mathcal{H}\left(J_{E}(E) \subset E^{\prime \prime}\right) .
$$

The Tsirelson-James space $T_{J^{*}}$ (see [2]) satisfies all the above hypotheses.

(b) If $E$ is reflexive, then under the hypotheses of Proposition 7 we obtain $\left(\mathcal{H}_{\omega}(E), \tau_{\omega}\right)^{\prime \prime}=\mathcal{H}(E)$. Moreover, if $\mathcal{P}_{\omega}\left({ }^{n} E\right)=\mathcal{P}\left({ }^{n} E\right)$, then $\mathcal{H}_{\omega}(E)=\mathcal{H}(E)$ and $\left(\mathcal{H}(E), \tau_{\omega}\right)$ is reflexive. The original Tsirelson space, $T^{*}$, satisfies these requirements and we recover a result in [1]. 
(c) If $x^{\prime \prime} \in E^{\prime \prime}, E$ is a Banach space, and

$$
\sum_{n=0}^{\infty}\left|J_{\mathcal{P}_{\omega}\left({ }^{n} E\right)}\left(\frac{\widehat{d}^{n} f(0)}{n !}\right)\left(x^{\prime \prime}\right)\right|<\infty
$$

for all $\sum_{n=0}^{\infty} \frac{\widehat{d}^{n} f(0)}{n !} \in \mathcal{H}_{\omega}(E)$, then the mapping

$$
\delta_{x^{\prime \prime}}: f \in \mathcal{H}_{\omega}(E) \longrightarrow \sum_{n=0}^{\infty}\left(J_{\mathcal{P}_{\omega}\left({ }^{n} E\right)}\right)\left(\frac{\widehat{d}^{n} f(0)}{n !}\right)\left(x^{\prime \prime}\right)
$$

defines a $\tau_{\delta}$-continuous multiplicative linear functional on $\mathcal{H}_{\omega}(E)$ (since it is the pointwise limit of a sequence of $\tau_{\delta}$-continuous linear functionals). If $E$ is separable and has the bounded approximation property, then $\tau_{\delta}=\tau_{\omega}$ and $\delta_{x^{\prime \prime}}$ is $\tau_{\omega}$ continuous on $\mathcal{H}_{\omega}(E)$. Moreover, using powers of $f$ and neighbourhoods of a compact set we see that $\delta_{x^{\prime \prime}}$ is $\tau_{0}$ continuous on $\mathcal{H}_{\omega}(E)$. Thus, in contrast to the case for holomorphic functions of bounded type (see Proposition 2), we obtain the algebraic identity $\mathcal{H}_{\omega}(E)^{\prime \prime}=\mathcal{H}\left(E^{\prime \prime}\right)$ if and only if $E$ is reflexive.

(d) In [6] and [9] the approximation property on $E^{\prime \prime}$ is replaced by using the $\tau_{0^{-}}$ closure of $J_{\mathcal{P}_{\omega}\left({ }^{n} E\right)}\left(\mathcal{P}_{\omega}\left({ }^{n} E\right)\right)$ in $\mathcal{P}\left({ }^{n} E^{\prime \prime}\right)$. This may also be used to obtain a variation of some of the results presented here.

The author would like to thank Manolo Maestre for interesting and helpful remarks on the subject matter of this paper.

\section{REFERENCES}

[1] R. Alencar, R. M. Aron, S. Dineen, A reflexive space of holomorphic functions in infinitely many variables, Proc. Amer. Math. Soc., 90, 1984, 407-411. MR 85b:46050

[2] R. M. Aron, S. Dineen, Q-reflexive Banach spaces, Rocky Mountain J. Math., 27, 4, 1997, 1009-1025. MR 99h:46010

[3] C. Boyd, R. A. Ryan, Geometric theory of spaces of integral polynomials and symmetric tensor products, J. Funct. Anal., (to appear).

[4] D. Carando, V. Dimant, Duality in spaces of nuclear and integral polynomials, J. Math. Ann. Appl. 241, 2000, 107-121. CMP 2000:07

[5] S. Dineen, Complex Analysis on Infinite Dimensional Spaces, Monographs in Mathematics, Springer Verlag (1999). CMP 99:17

[6] P. Galindo, M. Maestre, P. Rueda, Biduality in spaces of holomorphic functions, Math. Scand., 86, 2000, 5-16. CMP 2000:07

[7] M. González, Remarks on Q-reflexive spaces, Proc. Royal Irish Acad. Sect. A, 96, 2, 1996, 195-201. MR 99f:46024

[8] J. Horváth, Topological Vector Spaces and Distributions, Vol. I, Addison-Wesley, Reading, Massachusetts, 1996. MR 34:4863

[9] J. Mujica, M. Valdivia, Holomorphic germs on Tsirelson's space, Proc. Amer. Math. Soc.,123, 5, 1995, 1379-1386. MR 95f:46074

[10] L. Nachbin, Topological vector spaces of continuous functions, Proc. Nat. Acad. Sci. U.S.A., 40, 1954, 471-474. MR 16:156h

[11] T. Shirota, On locally convex vector spaces of continuous functions, Proc. Japan Acad. Ser. A, Math.Sci. 30, 1954, 294-298. MR 16:1030d

Department of Mathematics, University College Dublin, Belfield, Dublin 4, Ireland E-mail address: sean.dineen@ucd.ie 